\title{
Prevalence of Overweight/Obesity and Associated Factors Among Under-Five Children in Ethiopia: Evidence From the 2019 Ethiopia Mini Demographic and Health Survey (Emdhs); A Multilevel Analysis
}

Mathewos Alemu Gebremichael ( $\square$ alemumathewos2017@gmail.com )

Arba Minch University

Melkamu Merid Mengesha

Arba Minch University

Samuel Hailegebrea'l

Arba Minch University

Hanan Abdulkadir

Arba Minch University

Biruk Bogale Wolde

Mizan Tepi University

\section{Research Article}

Keywords: Under-five children, Overweight/obesity, Prevalence, Ethiopia

Posted Date: August 19th, 2021

DOl: https://doi.org/10.21203/rs.3.rs-724918/v1

License: (c) (1) This work is licensed under a Creative Commons Attribution 4.0 International License.

Read Full License 
1 Prevalence of overweight/obesity and associated factors among under-five children

2 in Ethiopia: Evidence from the 2019 Ethiopia Mini Demographic and Health Survey (EMDHS); A Multilevel Analysis

4 Mathewos Alemu Gebremichael ${ }^{1 *}$, Melkamu Merid Mengesha ${ }^{1}$, Samuel Hailegebrea' ${ }^{1}$, Hanan

$5 \quad$ Abdulkadir ${ }^{1}$, Biruk Bogale Wolde $^{2}$

$6{ }^{1}$ School of Public Health, College of Medicine and Health Sciences, Arba Minch University, 7 Arba Minch, Ethiopia.

$8 \quad{ }^{2}$ Department of Epidemiology and Biostatistics, School of Public Health, College of Medicine 9 and Health Sciences, Mizan Tepi University, Mizan Aman, Ethiopia. *Corresponding author: P.O. Box: 021, Arba Minch University, Arba Minch, Ethiopia Tel: +251917784364 , E-mail: alemumathewos2017@gmail.com/mathewos.alemu@ amu.edu.et

\section{Abstract}

Background: Childhood overweight and obesity are emerging public health challenges of the $21^{\text {st }}$ century. There was a $24 \%$ increase in the number of overweight children under the age of five years in low-income countries. Despite the significant risk of childhood overweight/obesity for non-communicable diseases, premature death, disability, and reproductive disorders in their adult life, little attention has been given. Therefore, we aimed to assess the prevalence of overweight/obesity and associated factors among under-five children.

Methods: This study was conducted using data from a nationally representative sample of the 2019 Ethiopia Mini Demographic and Health Survey (EMDHS). The Mini EDHS was a community-based cross-sectional study that covered all the administrative regions of Ethiopia.

The data collection was conducted between March 21, 2019, to June 28, 2019. Both descriptive 
and analytic findings were produced using STATA version 14. For associated factors, a multilevel binary logistic regression model was fitted to account for the hierarchical nature of the data. Adjusted odds ratio (aOR) with $95 \%$ confidence interval (CI) was reported to show the strength of association and statistical significance.

Results: A total 5,164 under-five children were included in this study. The overall prevalence of overweight/obesity was $2.14 \%(95 \% \mathrm{CI} ; 1.74-2.53)$ (more than two standard deviations (+2SD) above the median of the reference population) based on the body mass index (BMI) Zscore. The odds of overweight/obesity was higher among children aged less than 6 months $(\mathrm{aOR}=5.19 ;$ 95\%CI: 2.98-9.04), 6-24 months (aOR $=1.97$; 95\%CI: $1.18-3.29)$, delivered by caesarean section $(\mathrm{aOR}=1.75 ; 95 \% \mathrm{CI}$ : $1.84-3.65)$, living in Addis Ababa city $(\mathrm{aOR}=2.16$; 95\% CI: $1.59-7.81)$, Oromia region $(\mathrm{aOR}=1.93$; 95CI: $1.71-5.24)$, having mothers with the age 40-49 years $(\mathrm{aOR}=3.91 ; 95 \% \mathrm{CI}: 1.90-16.92)$, uses traditional contraceptive methods $(\mathrm{aOR}=$ 2.63; 95\%CI: $1.66-10.47)$, and households headed by male $(\mathrm{aOR}=1.71 ; 95 \% \mathrm{CI}: 1.84-3.48)$.

Conclusion: This study showed that childhood overweight/obesity is the problem in Ethiopia. There was an interplay of several factors that affect childhood obesity including child factors, maternal socio-demography, and healthcare utilization, and geography of residence. Therefore, strategies to reduce childhood overweight and obesity should consider a multitude of contributing factors.

Keywords: Under-five children, Overweight/obesity, Prevalence, Ethiopia

\section{Background}

According to the world health organization (WHO), overweight and obesity are defined as abnormal or excessive fat accumulation that can impair health (1). In 2019 and 2020, an estimated 38.2 and 39 million children under the age of 5 years were overweight or obese, 
respectively (1). Although childhood overweight and obesity have been considered the problems of high-income countries, are now on the rise in low- and middle-income countries. While these countries continue to deal with the problems of infectious diseases and undernutrition, childhood overweight and obesity are "double burdens" and the most serious public health challenges of the twenty-first century. Since 2000, the number of overweight children under the age of 5 has increased by nearly $24 \%$ in Africa $(1,2)$.

The number of overweight and obese children under the age of five has nearly doubled from 5.4 million in 1990 to 10.3 million in 2014, in Africa (3). According to 26 Demographic and Health, Surveys carried out in SSA since 2010, overweight and obesity in the children within the age group of $0-59$ months was reported $6.8 \%$ (4). Different studies suggest that childhood overweight and obesity in sub-Saharan Africa (SSA) is likely to be worsened by the current transition in nutrition and physical activity that is characterized by increased use of energy-saving devices, availability of cheap high calorie-dense foods, and limited participation in physical activity generally (5).

In low-income Sub-Saharan countries including Ethiopia, childhood obesity is not yet perceived as an emerging health issue and receives little attention. According to the annual report of UNICEF in 2017, there was an overall increment of the prevalence of overweight among children from 1.7 to $3.6 \%$ in Ethiopia (6). According to 26 Demographic and Health Surveys carried out in SSA since 2010, it was reported 3\% in Ethiopia (4). Although many studies have been conducted on the undernutrition of under-five children in Ethiopia, few studies reported that the combined prevalence of childhood obesity and overweight which was $10.7 \%$ and $3.4 \%, 7.3 \%$ for obesity and overweight, respectively in Hawassa town, and $13.8 \%$ in Gondar town and the pooled prevalence among children and adolescents in Ethiopia 11.30 (7-9). 
Childhood overweight and obesity are linked to more deaths than underweight and are associated with a higher chance of breathing difficulties, increased risk of fractures, hypertension, early markers of cardiovascular disease, insulin resistance, psychological effects, and adulthood obesity, premature death, and disability and resulting in an increased risk of noncommunicable diseases and reproductive disorders later in their life $(1,10)$.

Previous studies have reported many factors that were associated with overweight and obesity in under the age of five children. These are socioeconomic status, maternal education level, marital status, smoking during pregnancy, sex of the child, birth weight and the child's birth rank, residence, age of the child, body mass index (BMI) of parents, high dietary diversity, consumption of sweet food, time spent in watching television $>2 \mathrm{hr} /$ day, living in the grass field area, religion $(7,11-13)$.

Despite the rising of childhood overweight and obesity in Ethiopia, there is a paucity of information with robust statistical analysis. Therefore, this study aimed to assess the prevalence of overweight/obesity and determinants among under-five-aged children by using the current Ethiopia demographic and health survey data (EDHS). Thus, this finding could be input in designing effective preventive strategies to alleviate the rising burden of early childhood overweight/obesity and its consequential morbidity and mortality in children and later adulthood life.

\section{Methods and Materials}

\section{Study setting, design, and population}

This study used the 2019 Ethiopian Mini-EDHS data which was collected by the Ethiopian Public Health Institute (EPHI), in partnership with the Central Statistical Agency (CSA) and the Federal Ministry of Health (FMOH). Ethiopia is located in North-eastern Africa between $3^{0}$ and $15^{\circ}$ North latitudes and $33^{\circ} 48^{0}$ and East longitudes. The country has nine regional states and two city administrations (Addis Ababa and Diredawa) $(13,14)$. The survey was conducted 
in a nationally representative sample and provided estimates at national and regional levels for rural and urban areas (15).

A community-based cross-sectional study was conducted and data collection was done from March 21, 2019, to June 28, 2019. In this survey, 8,663 households were included and, data were collected from 5,753 children $<60$ months, 8,855 women of reproductive age (age 1549) from and 40,659 household members (15).

A stratified two-stage cluster sampling technique was applied. In the first stage, a total of 305 enumeration areas (EAs) (93 in urban areas and 212 in rural areas) were selected with probability proportional to EA size (based on the 2019 EPHC frame). In the second stage, a fixed number of 30 households per cluster were selected with an equal probability systematic selection from the newly created household listing. From all selected households, height and weight measurements were collected from children age 0-59 months, and women age 15-49 were interviewed using the Woman's Questionnaire (15).

\section{Data source}

Before downloading the data, an approval letter was obtained from the DHS and then the data set was downloaded from the DHS website (http://www.measuredhs.com). The kids' record data set was used from the downloaded data sets. Anthropometric data of under-five children and various pertinent socio-demographic variables were extracted from the data sets. The nutritional status of U5 children was measured using the weight-for-height (WFH) index. The WFH index was later categorized into normal weight (above minus two standard deviations $(-2 \mathrm{SD})$ and below plus two standard deviations (+2 SD) from the median of the reference population), underweight (below minus two standard deviations (-2 SD) from the median of the reference population) and overweight and obese (more than two standard deviations $(+2$ SD) above the median of the reference population) based on the BMI Z-score $(1,12,16)$.

\section{Study variables}


The outcome variable for this study was overweight/obesity in under-five children and it was coded as "1", whereas those children who were categorized as underweight, and normal were coded as "0". The independent variables were: Individual-level factors such as child age, child sex, birth order, twin child, delivered by caesarean section, mother's education status, current age, marital status, child ever born, age of mother at first birth, number of household members, sex of head of households, water source, toilet facility, wealth index, and contraceptive use. The community-level factors such as residence, region, and the altitude of clusters.

\section{Data management and statistical analysis}

Before analysis, we cleaned, categorized, and recorded different variables using STATA version 14. A total of 5,164 under-five children were included in this analysis after excluding those children whose BMI z-scores were missing or were recorded as out of plausible limits" or "Flagged cases", as their values were unusable. These data were recorded in the database under special codes which corresponded either to responses that were considered inconsistent with other responses in the questionnaire and thought to be probably an error or to responses whose value was "Don't know" $(12,16)$.

Both descriptive such as frequencies and proportions and analytic statistics were computed. A weighted analysis was done to compensate for the unequal probability of selection between the strata due to the non-proportional allocation of samples to different regions, places of residence, and non-response rate among study participants (15). Since demographic and health survey (DHS) data had a clustering and hierarchical nature. The individual-level factors (level 1) were nested within communities (level 2). A two-level mixed-effect logistic regression model was fitted to estimate both the independent (fixed) effect of the explanatory variables and the community-level (random) effect on our dependent variable childhood overweight/obesity (14). The rate of childhood overweight/obesity varies from cluster to cluster, a cluster-level random intercept was introduced in the mixed logit model. The within- 
cluster correlation was measured using intracluster correlation (ICC) which was $21.6 \%$ which indicated that the variations of overweight/obesity in under-five children were attributable to the difference at cluster level factors. The likelihood ratio test was applied to test the significance of the variance of random intercept. Adjusted odds ratio with corresponding 95\% confidence level was computed and reported to show the strength of the association and its significance. Variables having a p-value less than 0.05 were considered as having a significant association with the outcome variable. The deviance information criteria (DIC) was used to check the model goodness of fit.

\section{Results}

\section{Socio-demographic characteristics}

A total of 5,164 under-five children were included in the analysis. About $57.54 \%$ of children were found between the ages of 25 to 59 months and in a balanced male-to-female proportion where $50.87 \%$ were boys. A majority $75.07 \%$ were rural residents and $39.82 \%$ were from the Oromia region. Only $2.31 \%$ and $5.16 \%$ of children were twin and delivered by caesarean section, respectively (Table 1).

\section{Household and maternal characteristics}

From mothers in the reproductive age, the majority $22.30 \%$ were in the age category of $30-34$ years, the last $1.81 \%$ were in the age category of $45-49$ years. At the first birth, about $42.07 \%$ of women were under the age of 18 years. Regarding the education status of mothers, the majority $53.92 \%$ had no formal education followed by primary, secondary, and above $35.19 \%$, $7.29 \%$, and $3.60 \%$, respectively. About $86.98 \%$ of heads of households were male and the majority $23.57 \%$ of households were in the poorest wealth index rank (Table 2). 

had overweight/obesity. The majority $(90.44 \%)$ of under-five children were in the normal range of weight for height SD scores (Z-scores), $7.42 \%$ underweight and $2.14 \%$ overweight/obesity (Figure 1).

\section{Model building}

175 In this analysis, four models were fitted. The first model was the null model (Model 0) 176 containing no independent variables which were used to check the variability of overweight/obesity in the community and provide evidence to assess random effect using the intraclass correlation coefficient (ICC). The ICC in the null model was 0.21 , which means about $21.60 \%$ of the variations of overweight/obesity in the under-five-aged children were attributable to the difference at cluster level or community-level factors. The second (Model I) contains individual-level variables and the third (Model II) contains community-level variables. Both individual and community level variables were fitted simultaneously with the outcome variable in the fourth model (Model III). The model comparison was done using deviance and the fourth model with the lowest deviance (831.10) was selected as the best-fitted model (Table 3).

Table 3: Random effect and model comparison for factors associated with childhood overweight /obesity.

\begin{tabular}{|l|l|l|l|l|}
\hline Measures & Null model & Model I & Model II & Model III \\
\hline $\begin{array}{l}\text { Intraclass correlation } \\
\text { coefficient (ICC) }\end{array}$ & $21.60 \%$ & $18.54 \%$ & $16.35 \%$ & $15.15 \%$ \\
\hline Intercept & $0.90(0.42-1.92)$ & $0.75(0.32-1.73)$ & $0.64(0.26-1.62)$ & $0.59(0.21-1.63)$ \\
\hline $\begin{array}{l}\text { Akaike information } \\
\text { criterion (AIC) }\end{array}$ & 913.24 & 893.77 & 915.84 & 903.10 \\
\hline \begin{tabular}{l} 
Model fitness \\
\multicolumn{1}{c|}{ Log-likelihood }
\end{tabular} & -454.62 & -422.89 & -443.92 & -415.55 \\
\hline Deviance & 909.24 & 845.78 & 887.84 & 831.10 \\
\hline
\end{tabular}




\section{Factors associated with overweight/obesity}

In the bivariable multilevel binary logistic regression analysis, child age, child sex, current maternal age, maternal age at first birth, maternal educational level, sex of household head, child ever born, household wealth index, contraceptive use, child delivered by caesarean section, household toilet facility, residence, region and altitude of the cluster were associated with childhood overweight/obesity ( $p$-value $<0.25$ ). In the multivariable multilevel binary logistic regression analysis child age, maternal age, sex of household head, contraceptive use, child delivered by caesarean section, and region were significantly associated with under-five children overweight/obesity (p-value < 0.05) (Table 4).

Children in the age category of fewer than six months were 5.19 times more likely to be overweight/obese as compared to those children 25-59 months ( $\mathrm{aOR}=5.19 ; 95 \% \mathrm{CI}, 2.98-9.04)$. Similarly, children in the age group of $6-24$ months were 1.97 times more likely to be overweight/obese as compared to those children 25-59 months $\quad(\mathrm{aOR}=1.97 ; 95 \% \mathrm{CI}, 1.18$ 3.29). Maternal age was significantly associated with the nutritional status of under-five children. Children whose mothers age 40-49 years were 3.91 times more likely to be overweight/obese as compared to the children with maternal age from 15 -19 years $(\mathrm{aOR}=$ 3.91; 95\%CI, 1.90-16.92). The odds of overweight/obesity was higher in those children whose household head was male. Those children in the household headed by males had 1.71 times higher odds of overweight/obesity when compared to those children in the household headed by a female $(\mathrm{aOR}=1.71 ; 95 \% \mathrm{CI}, 1.84-3.48)$. Contraceptive use was another factor significantly associated with childhood overweight and obesity. Under-five children whose mother used traditional contraceptive methods were 2.63 times more likely to have overweight/obesity when compared to those children whose mother used modern contraceptive methods $(\mathrm{aOR}=2.63 ; 95 \% \mathrm{CI}, 1.66-10.47)$. Furthermore, children delivered by caesarean 
section were 1.75 times more likely to be overweight/obese as compared to their counterparts $(\mathrm{aOR}=1.75 ; 95 \% \mathrm{CI}, 1.84-3.65)$. The risk of overweight/obesity was 2.16 times higher in children's lives in Addis Ababa administrative city as compared to children in Tigray region $(\mathrm{aOR}=2.16 ; 95 \% \mathrm{CI}, 1.59-7.81)$. Likewise, under-five children in the Oromia region were 1.93 times at higher risk of overweight/obesity as compared to children who live in the Tigray region $(\mathrm{aOR}=1.93 ;$ 95CI, 1.71-5.24) $($ Table 4).

Discussion

221

Even though the prevalence of childhood under-nutrition is decreasing in Ethiopia, overweight/obesity emerging as a public health problem (7). Hence, this study assessed the prevalence of overweight/obesity and associated factors among under-five children in Ethiopia. The prevalence of overweight/obesity in this study was $2.14 \%$ (95\% CI; $1.74-$ 2.53). The factors associated with childhood overweight/obesity were: age of children, living regions, delivery by caesarean section, age of mothers, contraceptive use of mothers, and sex of head of households.

The $2.14 \%$ magnitude of overweight/obesity was consistent with the study done in Bahir Dar town which was $6.9 \%$ (17). The current finding was lower than the findings of the study in different settings; $13.8 \%$ in Gondar (7), the combined prevalence was $10.7 \%$ in Hawassa with $7.3 \%$ overweight and $3.4 \%$ obesity (9) and $7 \%$ the global prevalence (18).

The prevalence of overweight/obesity in this study was also lower than a study conducted in Cameroon which was 8\% (19), in Kenya 20.3\% (20), in Sub-Saharan Africa 6.8\% (4) in Nigeria $23.6 \%$ (21). A study done in Iran reported that $11.8 \%$ overweight and $15 \%$ obese (22), in urban and rural Vietnam, $21.1 \%$ and $7.6 \%$, respectively (23). In Malawi, the combined prevalence of childhood overweight and obesity was $14.5 \%$ with the $8.7 \%$ overweight and $5.8 \%$ obesity (24), in Mozambique $11.9 \%$ (7.7\% overweight and $4.2 \%$ obesity) (24), and in Brazil, the overweight was $9.7 \%$ (25). In Iraq the combined prevalence of overweight and 
obesity was $11.2 \%$ (7.6\% overweight and $3.6 \%$ obesity) (26), in Iran $35.7 \%$ (12\% overweight and $23.7 \%$ obesity) (27), and in Brazil, it was $21.9 \%$ with the $14.4 \%$ overweight and $7.5 \%$ obese (28). The observed difference might be due to the variations in socio-cultural and socioeconomic status in dietary intake, lifestyle, and study time.

In the current study, the age of the child was significantly associated with childhood overweight/obesity. The odds of overweight and obesity was 5.19 times higher among children whose age group fewer than six months as compared to those children in the age group of 2559. Similarly, children in the age category of 6-24 months had 1.97 times higher odds of overweight and obesity when compared to those children in the age group of 25-59 months. This finding was in agreement with the study conducted in Gondar, Hawassa, Ethiopia, and Cameroon $(7,9,11,29)$. In these study findings, younger children had a higher risk of becoming overweight or obese than their older comparative age groups. The possible reason might be that when the age of the child increases, the chance of joining the school would increase and this may attribute to physical activity, which would lead to increased metabolic activity and energy requirements. The other possible explanation maybe during the early childhood period physiologically the percentage of body fat decreases and muscle tissue increases and children get thinner. This study report suggests that age-specific nutritional counselling programs and strategies during childhood are necessary.

The present study showed that maternal age was significantly associated with the overweight and obesity of under-five children. The odds of under-five children overweight and obesity was 3.91 times higher among children whose maternal age was 40-49 years as compared to those children whose maternal age from 15-19 years. The possible explanation might be as the age of mothers increase, the body mass index (BMI) also does increase. Studies reported that there is a positive association between the BMI of mothers and the overweight and obesity of children (30). For this association, the following reasons were suggested: these include 
inheritance of genes that enhance susceptibility, and the behaviors of poor eating habits (31, $32)$.

This study revealed that the risk of overweight and obesity was higher in those children whose households were headed by males. Those children in the household headed by males had 1.71 times higher odds of overweight and obesity when compared to those children in the household headed by a female. This might be because of the food security status of the household. Pooled estimate from Systematic-review and meta-analysis in Ethiopia indicated that the households headed by males had two-fold times higher odds of food security when compared to the female-headed households (33). Hence, those households headed by males might encourage their children to take energy-dense foods that are high in fat and sugars and change in modes of transportation, which increases sedentary life this leads to physical inactivity.

Contraceptive use was another factor significantly associated with childhood overweight and obesity. Under-five children whose mother used traditional contraceptive methods were 2.63 times more likely to have overweight and obesity when compared to those children whose mother used modern contraceptive methods. The possible reason might be those mothers who use traditional contraceptive methods may have low awareness about the effects of energydense foods that are high in fat and sugars, physical inactivity due to sedentary lifestyles. However, the comparative category of mothers of under-five children who use modern contraceptive methods may have good awareness because of the variety of health education during attending for the family planning schedule in health facilities.

Furthermore, children delivered by caesarean section were 1.75 times more likely to be overweight and obese as compared to their counterparts. The association between caesarean delivery and childhood overweight and obesity is not clear. However, the possible reason can be the weight of the child at birth. Studies showed that child overweight at birth was a risk for 
caesarean delivery as well as for overweight and obesity in later life $(34,35)$. The other suggested possible mechanism was the hygiene hypothesis $(36,37)$. In this hypothesis, caesarean delivery deprives the chance for the new-born baby to be exposed to maternal vaginal faeces, the bacteria from which are a major source for the intestinal bacteria of the new-born. As a result, as compared with those born vaginally, new-borns delivered by caesarean section had fewer intestinal Bifidobacteria and Bacteroides both of which were reported to be protective factors against later obesity $(38,39)$. The association was also supported by indirect epidemiological evidence, in addition to hygiene theory (40). Caesarean delivery was associated with a lower umbilical leptin concentration and it reduces the rate of early breastfeeding both of which were reported to be associated with an increased risk of later overweight and obesity (41-44).

The risk of overweight and obesity was 2.16 times higher in children living in Addis Ababa administrative city as compared to children in the Tigray region. Likewise, under-five children living in the Oromia region were 1.93 times at higher risk of overweight and obesity as compared to children living in the Tigray region. This variation might be due to differences in the habit of food eating, and sedentary lifestyle. Because of the better socio-economic status of the population, and the better access to high-calorie diets in Addis Ababa compared to the Tigray regions of the country.

\section{Strengths and limitations of the study}

The study used a large survey and nationally representative data including regional variation, factors at individual and community levels. However, there were some limitations to this study. The cross-sectional nature of the data prevents causality from being inferred between the independent and dependent variables. This study only focused on the specific factors of overweight and obesity but did not include missed variables such as dietary intake, feeding habits of the children, maternal nutritional status, and the weight of the child at birth these 
might be the residual confounding factors. This study assessed the information from the five years before the survey period; it might be prone to recall bias, particularly for age or other retrospective data relying on memory of past events.

\section{Conclusion}

This study showed that the prevalence of overweight/obesity among under-five was $2.14 \%$ in Ethiopia. Age of children, living regions, delivery by caesarean section, age of mothers, contraceptive use of mothers, and sex of head of households were statistically associated factors with childhood overweight/obesity. Therefore, giving emphasis to children with these above characteristics and formulating preventive programs and policies during children's early years are highly recommended.

\section{Abbreviations:}

aOR, adjusted odds ratio; AIC, Akaike information criterion; BMI, body mass index CI, confidence interval; cOR, crude odds ratio CSA, central statistics agency; EA, enumeration areas; EMDHS, Ethiopian mini demographic and health survey; EPHI, Ethiopian public health institute; FMoH, Federal ministry of health; ICC, Intraclass correlation coefficient; PHC, Population and housing census; SSA, Sub-Saharan Africa.

\section{Ethics approval and consent to participate}

The primary researchers of the 2019 Ethiopia Mini Demographic Health Survey obtained ethical clearance, and there was no need for ethical clearance for this secondary analysis. However, we obtained an authorization letter on May 28, 2021, to download the EMDHS-2019 data set from the http://www.measuredhs.com website. The obtained data were treated strictly confidential and were used only for this study. 
337 The Mini Demographic and Health Surveys (DHS) 2019 data set is available at 338 https://dhsprogram.com/data/availabledatasets.cfm. The DHS Program is authorized to 339 distribute, at no cost, unrestricted survey data files for legitimate academic research. 340 Registration is a prerequisite for access to data. The data sets are publicly available to all 341 registered users and downloaded from the website

\section{Competing interests}

343 The authors declare that they have no competing interests

\section{$344 \quad$ Funding}

345 The author(s) received no funding for this work.

\section{Authors' contributions}

347 MAG involved in the study from the conception to designing, acquisition of data, analysis, and 348 interpretation, and drafting of the manuscript. MMM, $\mathrm{SH}, \mathrm{HA}$, and $\mathrm{BBW}$ involved in the 349 interpretation and drafting of the manuscript. All authors read and approved the final 350 manuscript and agreed to be personally accountable for the author's contributions and to ensure 351 that questions related to the accuracy or integrity of any part of this study.

\section{Acknowledgments}

353 We would like to thank the Measure Demographic and health survey (DHS) international 354 program for providing the data set

\section{$355 \quad$ References}

356 1. WHO. Key facts about obesity and overweight. 9 June 2021 [cited 2021 June 17].

357 Available from: https://www.who.int/news-room/fact-sheets/detail/obesity-and-overweight.

358 2. UNICEF W, World Bank. Levels and trends in child malnutrition: UNICEF-WHO359 World Bank joint child malnutrition estimates. New York, Geneva, Washington DC: UNICEF, 
WHO, World Bank. 2015 [cited 2021 June 22]. Available from: https://www.who.int/nutgrowthdb/jme_brochure2016.pdf. 3. WHO. Commission presents its fnal report, calling for high-level action to address major health challenge 25 January 2016 [17 Jun 2021]. Available from: https://www.who.int/ end-childhood-obesity/news/launch-fnal-report/en/.

4. Gebremedhin S. Prevalence and differentials of overweight and obesity in preschool children in Sub-Saharan Africa. BMJ open. 2015;5(12):e009005.

5. Onywera VO. Childhood obesity and physical inactivity threat in Africa: strategies for a healthy future. Global health promotion. 2010;17(2_suppl):45-6.

6. UNICEF W, World Bank. Annual Report 2016, Ethiopia. www.unicef.org/about/annualreport/fles/Ethiopia_2016_COAR.pdf. 2018 [Accessed 17 Jun 2021.].

7. Sorrie MB YM, GebreMichael, TGGyorgis. Overweight/Obesity and associated factors among preschool children in Gondar City, Northwest Ethiopia: A cross-sectional study. . PLoS ONE 2017;12(8).

8. Gebrie A, Alebel A, Zegeye A, Tesfaye B, Ferede A. Prevalence and associated factors of overweight/obesity among children and adolescents in Ethiopia: a systematic review and meta-analysis. BMC obesity. 2018;5(1):1-12.

9. Wolde T, Belachew T. Prevalence and determinant factors of overweight and obesity among preschool children living in Hawassa City, South Ethiopia. Prevalence. 2014;29.

10. World $\mathrm{OH}$. Childhood overweight and obesity on the rise, 2010. (WWW document). 2010 [Accessed 17 July 2021]. Available from: http://www.who.int.

E. Prevalence and risk factors of overweight and obesity among children aged 6-59 months in 
Cameroon: a multistage, stratified cluster sampling nationwide survey. PloS one. 2015;10(12):e0143215.

12. Weldearegay HG, Gebrehiwot TG, Abrha MW, Mulugeta A. Overweight and obesity among children under five in Ethiopia: further analysis of 2016 national demographic health survey: a case control study. BMC research notes. 2019;12(1):1-6.

13. Toselli S, Zaccagni L, Celenza F, Albertini A, Gualdi-Russo E. Risk factors of overweight and obesity among preschool children with different ethnic background. Endocrine. 2015;49(3):717-25.

14. Wolde HF, Gonete KA, Akalu TY, Baraki AG, Lakew AM. Factors affecting neonatal mortality in the general population: evidence from the 2016 Ethiopian Demographic and Health Survey (EDHS)—multilevel analysis. BMC research notes. 2019;12(1):1-6.

15. Ethiopian Public Health Institute (EPHI) [Ethiopia] and ICF. Ethiopia Mini ICF 2021 [cited 2021 June 22].

16. Guide to DHS Statistics DHS-7: Nutrition of Children and Adults [cited 2021 June 22]. Available from: https://dhsprogram.com/.

17. Tadesse Y, Derso T, Alene KA, Wassie MM. Prevalence and factors associated with overweight and obesity among private kindergarten school children in Bahirdar Town, Northwest Ethiopia: a cross-sectional study. BMC research notes. 2017;10(1):1-6.

18. Wang Y, Lim H. The global childhood obesity epidemic and the association between socioeconomic status and childhood obesity. Int Rev Psychiatry. 2012;24(3):176-88. Multistage, Stratified Cluster Sampling Nationwide Survey. . PLoS ONE 2015;10(12). 
20. Wandia FB, Ettyang G, Mbagaya G. Prevalence of and factors associated with

409

410

411

412

413

414

415

416

417

418

419

420

421

422

423

424

425

426

427

428

429

430

overweight and obesity among nursery school children aged 3-6 years in Eldoret Municipality.

African Journal of Food, Agriculture, Nutrition and Development. 2014;14(5):2057-71.

21. Mezie-Okoye M, Alex-Hart B. Overweight and obesity among preschool children in port Harcourt, Nigeria. Pakistan Journal of Nutrition. 2015;14(4):209.

22. Hajian-Tilaki K, Heidari B. Childhood obesity, overweight, socio-demographic and lifestyle determinants among preschool children in Babol, northern Iran. Iranian journal of public health. 2013;42(11):1283.

23. Do LM, Tran TK, Eriksson B, Petzold M, Nguyen CT, Ascher H. Preschool overweight and obesity in urban and rural Vietnam: differences in prevalence and associated factors. Global health action. 2015;8(1):28615.

24. Gebremedhin S.Prevalence and differentials of overweight and obesity in preschool children in Sub Saharan Africa. BMJ Open, 2015. 5(12): p. e009005. https://doi.org/10.1136/bmjopen-2015-009005PMID: 26700276.

25. Risia Cristina Egito de Menezes, Pedro Israel Cabral de Lira, Souza Oliveira Juliana,Sa Leal Vanessa,Sandra Cristina da Silva Santana,Filho Malaquias Batista, et al.Prevalence and determinants of over weight in preschool children. Jornal de Pediatria, 2011. 87(3): p. 231237. https://doi.org/doi:10.2223/JPED.2092 PMID: 21566856.

26. Musa Wafaa Ali, Hassan Meaad K.. overweight and obesity among preschool children in basrah. themedical journal of basrah university, MJBU, 2010. 28(1).

27. Hajian-Tilaki K,Heidari B. Childhood Obesity, Overweight, Socio-Demographic and Life Style Determi nants among Preschool Children in Babol, Northern Iran. Iran J Public Health, 2013. 42(11): p. 1283-91. PMID: 26171341. 
28. Schucha Ilaine, de Castrob Teresa G., de Vasconcelosc Francisco de A.G., Goldanie

432

433

434

435

436

437

438

439

440

441

442

443

444

445

446

447

448

449

450

451

452

453

454

Marcelo Z.,Dutrad Carmem L.C.. Excess weight in preschoolers: prevalence and associated factors. J Pediatr (RioJ), 2013. 89: p. 179-88.

29. al We. Overweight and obesity among children under fve in Ethiopia: further analysis of 2016 national demographic health survey: a case control study. BMC Res Notes (2019) 12:716 2019;12(716).

30. Janjua NZ, Mahmood B, Islam MA, Goldenberg RL. Maternal and early childhood risk factors for overweight and obesity among low-income predominantly black children at age five years: a prospective cohort study. Journal of obesity. 2012;2012.

31. Chagnon YC, Rankinen T, Snyder EE, Weisnagel SJ, Pérusse L, Bouchard C. The human obesity gene map: the 2002 update. Obesity research. 2003;11(3):313-67.

32. Whitaker RC, Dietz WH. Role of the prenatal environment in the development of obesity. The Journal of pediatrics. 1998;132(5):768-76.

33. Negesse A, Jara D, Temesgen H, Dessie G, Getaneh T, Mulugeta H, et al. The impact of being of the female gender for household head on the prevalence of food insecurity in Ethiopia: a systematic-review and meta-analysis. Public Health Reviews. 2020;41:1-14.

34. Kelly NM, Keane JV, Gallimore RB, Bick D, Tribe RM. Neonatal weight loss and gain patterns in caesarean section born infants: integrative systematic review. Maternal \& child nutrition. 2020;16(2):e12914.

35. Ye R, Pei L, Ren A, Zhang Y, Zheng X, Liu J-m. Birth weight, maternal body mass index, and early childhood growth: a prospective birth cohort study in China. Journal of epidemiology. 2010:1008250186-.

36. Neu J, Rushing J . Cesarean versus vaginal delivery: long-term infant outcomes and the hygiene hypothesis. Clin Perinatol 2011; 38: 321-331. 

microbiota metabolic interactions. Science 2012; 336: 1262-1267.

457

458

459

460

461

462

463

464

465

466

467

468

469

470

471

472

473

38. Gronlund MM, Lehtonen OP, Eerola E, Kero P . Fecal microflora in healthy infants born by different methods of delivery: permanent changes in intestinal flora after cesarean delivery. J Pediatr Gastroenterol Nutr 1999; 28: 19-25.

39. Kalliomäki M, Carmen Collado M, Salminen S, Isolauri E. Early differences in fecal microbiota composition in children may predict overweight. The American journal of clinical nutrition. 2008;87(3):534-8.

40. Li H-t, Zhou Y-b, Liu J-m. Cesarean section might moderately increase offspring obesity risk. The American journal of clinical nutrition. 2012;96(1):215-6.

41. Yoshimitsu N, Douchi T, Kamio M, Nagata Y. Differences in umbilical venous and arterial leptin levels by mode of delivery. Obstetrics \& Gynecology. 2000;96(3):342-5.

42. Zanardo V, Svegliado G, Cavallin F, Giustardi A, Cosmi E, Litta P, et al. Elective cesarean delivery: does it have a negative effect on breastfeeding? Birth. 2010;37(4):275-9.

43. Armstrong J, Reilly JJ. Breastfeeding and lowering the risk of childhood obesity. The Lancet. 2002;359(9322):2003-4.

44. Mantzoros CS, Rifas-Shiman SL, Williams CJ, Fargnoli JL, Kelesidis T, Gillman MW. Cord blood leptin and adiponectin as predictors of adiposity in children at 3 years of age: a prospective cohort study. Pediatrics. 2009;123(2):682-9. 


\section{Figures}

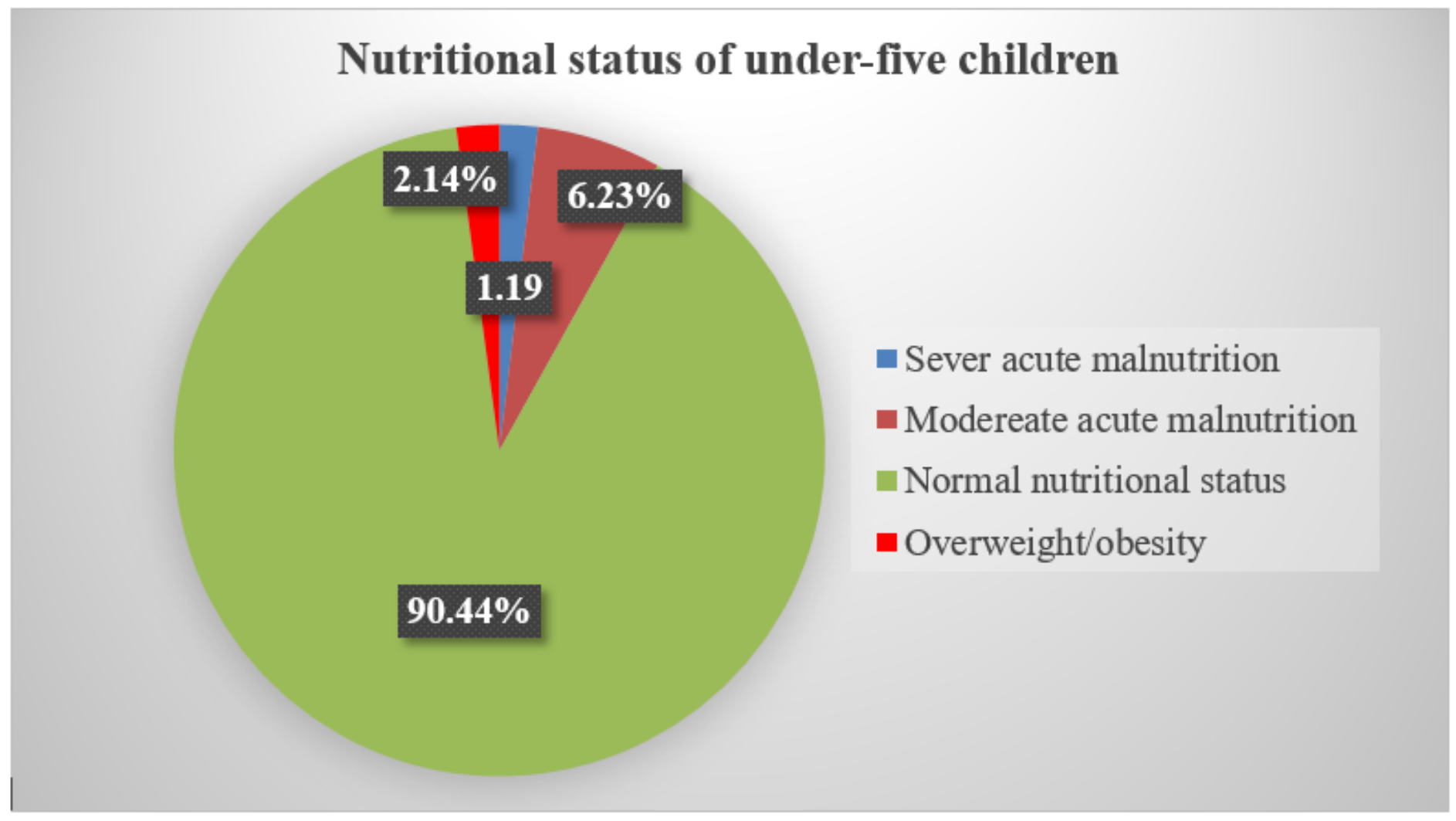

\section{Figure 1}

Nutritional status in under-five aged children in Ethiopia: Data from Ethiopia Mini Demographic and Health Survey (EMDHS 2019). 